\title{
Der Entwurf des neuen ungarischen Irrengesetzes (von Otto Freiherrn von Babarezi-Sehwartzer).
}

\author{
Mitgeteilt von \\ Priv.-Doz. Carl Hudovernig (Budapest).
}

(Eingegangen am 31. Januar 1911.)

Die mit Riesenschritten schreitende moderne klinische Psychiatrio brachte selbstverständlich auch eine rapide Entwicklung sämtlicher: Zweige psychiatrischer Hilfswissenschaften, namentlich der forensischen Psychiatrie, des psychiatrischen Anstaltswesens und der Irrenfürsorge mit sich. Gerade die letztere war bisher in fast sämtlichen Ländern derart stiefmütterlich bedacht, daß sich allerorts das Bedürfnis nach einer sowohl für die Irrenärzte als auch für die Kranken überaus erwünschten endgültigen und den heutigen Auffassungen und Anforderungen entsprechenden Regelung des administrativen Irrenwesens immer mehr fühlbar machte.

Auch in Ungarn empfanden die Psychiater seit langer Zeit, dab eine Regelung des Irrenwesens dringend nötig sei, denn eine einheit-liche Regelung, resp. gesetzliche Fassung der verschiedensten, meistt nur für konkrete Fälle ad hoc erlassenen Verordnungen fehlte in Ungarn ebenso wie in den meisten Kulturstaaten. Werböczis Jus Tripartitum, sowie einige spätere Gesetze bezogen sich zumeist nur auf die zivilund strafrechtlichen Verhältnisse der Irren. Erst das Gesetz XIV vom Jahre 1876 brachte eine Regelung der hygienischen Fragen und erstreckt sich in einem kurzen, aus 6 Paragraphen bestehenden Abschnitte auf den administrativen Teil des Irrenwesens. In einigen späteren ungarischen Gesetzen finden wir nur wenige und kurze Verfügungen, überdies "regelt" eine Unzahl von Ministerialverordnungen das ungarische Irrenwesen, Ministerialverordnungen, welche zumeist im Anschlusse an konkrete Fälle eigentlich nur ad hoc Verfügungen enthalten.

Diese Mißstände fühlte der ungarische Landessanitätsrat seit langem und entsandte aus diesem Grunde eine Reformkommission, welche den Vizepräsidenten des Senates, Otto Freiherrn von BabarcziSchwartzer mit der Ausarbeitung der Grundprinzipien eines Irren. gesetzes betraute. Frh. v. Babarczi-Schwartzer entwickelte dieselben vor dem 1902 abgehaltenen Landeskongresse der ungarischen Psychiater, und nachdem dieser die Grundprinzipien akzeptierte, stellte die er. 
wähnte Reformkommission die Grundzüge des Irrengesetzes definitiv fest, und auf Grund derselben arbeitete Frh. v. Babarczi-Schwartzer den Entwurf eines ungarischen Irrengesetzes aus, welcher nach Approbierung durch den Landessanitätsrat dem Ministerium des Inneren bereits im Jahre 1904 vorgelegt wurde. Verschiedene Umstände und die leidlichen politischen Verhältnisse in Ungarn haben die Gesetzwerdung des Entwurfes bisher verzögert, doch ist zu hoffen, daß die definitive Erledigung dieser Frage, welche der Verfasser des Gesetzentwurfes jüngst noch mit einigen Verfügungen über beschränkt zurechnungsfähige Individuen erweitert hat, nicht mehr so lange zögern wird.

Der Gesetzentwurf umfaßt 104 Paragraphen in 12 Abschnitten. Die wesentlichsten Verfügungen des Gesetzentwurfes, welche bei den allgemeinen Bestrebungen nach einheitlicher Regelung des Irrenwesens gewiß weitere Kreise interessieren dürften, sind die folgenden:

Das projektierte Gesetz ist ausschließlich administrativer, resp. verwaltungsrechtlicher Natur, wobei die straf- und zivilrechtlichen Angelegenheiten der Geisteskranken einer anderen gesetzlichen Regelung vorbehalten bleiben. An dieser Stelle wäre noch hervorzuheben, daß einzelne prinzipielle Fragen, welche der Gesetzentwurf vom verwaltungsrechtlichen Standpunkte in modernẹr Auffassung regelt, z. B. Verfügungen bezüglich Vormundschaft, im neuen BGB. bereits übernommen wurden.

Der erste Abschnitt, $\S 1-2$, spricht aus, daß die Leitung und Beaufsichtigung des Irrenwesens eine staatliche Aufgabe ist, und daß die Verfügungen dieses Gesetzes sich auf jede geistige Erkrankung beziehen, ohne Rücksicht auf Art und Dauer der Krankheit.

Im Motivenberichte des Gesetzentwurfes wird namentlich jene Frage aufgeworfen, ob eine Spezifizierung der Formen geistiger Erkrankungen wünschenswert und in präziser Weise möglich sei. Obwohl einige Irrengesetze, administrative, zivil- und strafrechtliche, spezielle Formen der geistigen Erkrankungen anführen, ist eine derartige gesetzliche Verfügung gerade in einem rein verwaltungsrechtlichen Gesetze nicht am Platze, weil ja die verschiedenen Einschränkungen der zivilrechtlichen Beziehungen bei „Geisteskrankheit und Schwachsinn“ erklärlich und gerechtfertigt sein mögen, aber nicht in einem administrativen Gesetze, um so weniger, weil wir noch immer nicht in der Lage sind, die verschiedenen Formen und Arten der geistigen Erkrankung klinisch präzis zu regeln.

Der zweite Abschnitt, $\S 3$-4, verfügt prinzipiell über die Unterbringung der Geisteskranken; während das Gesetz XIV vom Jahre 1876 als Kriterien der Anstaltsunterbringung neben Selbst- 
und Gemeingefährlichkeit auch die Frage der Heilbarkeit, also Notwendigkeit der Unterbringung in einer "Irrenheilanstalt" vorsah, beschränkt sich dieser Abschnitt des neuen Gesetzentwurfes bloß darauf, daß ohne Rücksicht auf die Frage der Heilbarkeit für die Anstaltsunterbringung ausschließlich die Frage der "Gefährlichkeit" maßgebend sei. In diesem Sinne verfügt der Gesetzentwurf, daß gefährliche Geisteskranke in Irrenanstalten, nicht gefährliche Geisteskranke in häuslicher, Familien- oder Gemeindepflege unterzubringen sind.

Der dritte Abschnitt, §5-30, regelt das Wesen der Irrenanstalten. Als solche wird jede Lokalität und Kolonie bezeichnet, in welcher nicht zur Familie des Inhabers gehörige Geisteskranke gepflegt werden. (Der Begriff der Familie wird in $\$ 35$ des Entwurfes präzisiert.) Auf Grund dieser prinzipiellen Bestimmung des Begriffes der Irrenanstalt wird es unmöglich gemacht, daß sich Unternehmungen, welche sich unter beliebiger Benennung mit Irrenpflege befassen, der staatlichen Überwachung entziehen; ebenso sind auch Geisteskrankenkolonien auf diese Weise in den Sammelbegriff der Irrenanstalten einbezogen worden. Der Entwurf unterscheidet öffentliche und private Irrenanstalten; öffentliche Anstalten sind jene, welche Staat, Kommune oder Gemeinde errichten oder unterhalten. Zur Errichtung privater Anstalten können bloß Ärete oder solche Orden resp. Wohlfahrtsvereine Erlaubnis erhalten, welche sich berufsmäßig resp. statutenmäßig mit Krankenpflege beschäftigen. Kommunale, Gemeinde- und Privatirrenanstalten können nur mit ministerieller Erlaubnis errichtet werden; nicht ungarischen Staatsbürgern, Personen, welche nicht geschäftsfähig sind oder unter Konkurs stehen, ferner wegen Verbrechen oder aus Gewinnsucht begangener Vergehen rechtskräftig verurteilt sind, kann die Erlaubnis nicht erteilt werden, ebensowenig solchen Personen, bei welchen der Verdacht eines Mißbrauches besteht. \$ 9-16 regeln die bei Ansuchen zur Anstaltserrichtung erforderlichen Formalitäten und technische, sowie Einrichtungsanforderungen. Die Irrenanstalt muß durch einen nach allen Richtungen verantwortlichen Direktor geleitet werden, welcher nur ein Arzt sein kann. Bei Direktoren und Oberärzten öffentlicher Anstalten ist psychiatrische Fachbildung und wenigstens zweijährige Anstaltstätigkeit erforderlich. Nach § 20 muß der Direktor einer privaten Heilanstalt dieselbe Qualifikation besitzen wie der Direktor einer öffentlichen Anstalt; überdies sind für ihn dieselben Bedingungen maßgebend wie für den Besitzer einer privaten Irrenanstalt. Aufnahme und Entlassung des ärztlichen Pflegeund Hilfspersonals der privaten Anstalten muß dem Direktor derselben überlassen sein. Nach $\S 23 \mathrm{muß}$ in jeder Irrenanstalt für je 100 Kranke ein Arzt, und für je 10 Kranke ein Pfleger bestellt sein; Abweichungen hievon dürfen nur mit ministerieller Erlaubnis statt- 
finden; sämtliche Pfleger und zumindest ein Arzt müssen im Anstaltsterritorium wohnen. Im Falle des Ablebens des Besitzers einer Privatanstalt übergeht die Anstalt auf die rechtmäßigen Erben resp. Nutznießer; private Anstalten können mit Genehmigung des Ministeriums des Inneren auch übertragen werden. Die Erlaubnis zur Errichtung, resp. Führung (nicht Leitung) kann zurückgezogen werden, wenn die Anstalt innerhalb zweier Jahre nicht eröffnet wurde, resp. der Betrieb zwei Jahre hindurch eingestellt bleibt; ferner in allen Fällen, welche die Genehmigung der Anstalt als unzulässig erscheinen lassen, ausgenommen den Fall, wenn der Besitzer seine Geschäftsfähigkeit einbüßt oder über ihn der Konkurs verhängt wird. Liegt der Grund zur Entziehung der Erlaubnis in der Person des Besitzers, so ist die Übertragung der Erlaubnis statthaft. Der Entwurf regelt ferner auch jene Bedingungen, unter welchen die Berechtigung zur Leitung einer Anstalt entzogen werden kann: in allen Fällen, welche die Berechtigung zur Leitung einer Anstalt ausschließen (s. oben), ferner wenn der Direktor einer Irrenanstalt wegen im Entwurfe vorgesehener Vergehen oder wegen anderer Vergehen innerhalb fünf Jahren strafrechtlich dreimal verurteilt worden ist. Bei Austritt des Direktors aus einer Anstalt erlischt seine Berechtigung zur Leitung einer Irrenanstalt.

Der vierte Abschnitt umfaßt $\S 31-42$ und regelt die Aufnahme in die Irrenanstalt. In diesem Abschnitte begegnen wir zwei wichtigen und prinzipiellen Neuerungen. Es sind dies erstens die essentielle Unterscheidung der „Krankenübernahme“ und der „Aufnahme" des Kranken in die Irrenanstalt. Die Übernahme des Kranken bedeutet das, daß auf Grund eines ärztlichen Zeugnisses das als geisteskrank bezeichnete Individuum in die Irrenanstalt übernommen wird, ohne jedoch, daß hierdurch für den Kranken irgendwelche Rechtsfolgen erwachsen, und ohne daß durch diese Übernahme seine Aufnahme in die Irrenanstalt als eine rechtskräftige Handlung von zivilrechtlichen Folgen erscheinen würde. Die Übernahme des Kranken wird erst dann zu einer rechtskräftigen Aufnahme, wenn das zuständige Bezirksgericht, welches von jeder Krankenübernahme sofort $\mathrm{zu}$ verständigen ist, die Geisteskrankheit durch einen Sachverständigen feststellen läßt, und auf Grund dieses Gutachtens die rechtskräftige Aufnahme des als geisteskrank Erkannten in die Irrenanstalt ausspricht. Diese Verfügungen waren z. T. auch im bisher gültigen Gesetz XIV von 1876 vorgesehen, indem die provisorische und endgültige Aufnahme unterschieden wurde, und die letztere bei Privatirrenanstalten erst nach einer gerichtlichen Untersuchung in Kraft trat. Doch war gerade diese Amtshandlung in Ungarn bisher gleichzeitig mit der Frage der Kuratelverhängung verknüpft, welcher Vorgang eigentlich auf einem Verkennen der verschiedenen diesbezüg- 
lichen Bestimmungen und Ministerialverordnungen beruhte, indem die durch das zuständige Gericht entsendeten Sachverständigen nicht nur das Bestehen der Geisteskrankheit, sondern sich auch über die Geschäftsfähigkeit des Kranken aussprachen, und das Gericht so die richterliche Feststellung der Geisteskrankheit gleichzeitig als erste Etappe des Kuratelverfahrens betrachtete. Nach den derzeitigen gesetzlichen Bestimmungen hat der Direktor einer Privatirrenanstalt die Aufnahme eines Geisteskranken innerhalb 24 Stunden dem zuständigen Gerichtshofe anzumelden, welcher die Geisteskrankheit durch Sachverständige feststellen läßt, während die staatlichen und Spitalsirrenanstalten nach einer gewissen Beobachtungsdauer der zuständigen Gerichtsbehörde Bericht erstatten, welche dann auf Grund dieses, die Geisteskrankheit feststellenden Berichtes (welcher die Rechtsgültigkeit eines Sachverständigen-Gutachtens besitzt) gleichzeitig das Verfahren bezüglich der Kuratelverhängung einleitet, ebenso wie bei Kranken in Privatirrenanstalten auf Grund des eingelangten Gutachtens der Sachverständigen. Demgegenüber trennt der vorliegende Entwurf in schärfster Weise die amtliche Feststellung der bestehenden Geisteskrankheit, welche bloß die endgültige, resp. rechtskräftige Aufnahme in die Irrenanstalt bedeutet, von dem Kuratelverfahren, und hierin liegt die zweite prinzipielle Neuerung des vorliegenden Gesetzentwurfes. Das Bezirksgericht spricht bloß die Berechtigung einer Internierung in der Irrenanstalt fest; die Kuratelverhängung erfolgt auf Grund eines separaten Ansuchens der kompetenten Angehörigen an den Gerichtshof, und erfolgt die Kuratelverhängung auf Grund eines Gutachtens der Gerichtssachverständigen. Auf diese Weise kann der Kranke wegen Geisteskrankheit in einer Irrenanstalt interniert sein, ohne daß hierdurch auch gleichzeitig das Kuratelverfahren eingeleitet werden würde.

Die detaillierten Bestimmungen dieses Abschnittes sind folgende: Die Übernahme von Geisteskranken in eine Irrenanstalt kann entweder über Aufforderung einer Behörde oder über schriftliches Ansuchen der Angehörigen erfolgen. (Die Modalitäten eines freiwilligen Eintrittes werden in einem eigenen Abschnitte geregelt.) Ohne Zeugnis eines in öffentlicher Anstellung befindlichen Arztes darf prinzipiell kein Geisteskranker aufgenommen werden; ist es aber evident, daß die Erwerbung eines solchen Zeugnisses unmöglich war, kann der Anstaltsdirektor den Kranken auf eigene Verantwortung übernehmen, jedoch muß in derartigen Fällen das fehlende Zeugnis innerhalb 48 Stunden beigebracht werden. Vom etwaigen behandelnden Arzte ist eine Krankengeschichte einzuholen. Das ärztliche Zeugnis muß neben Personaldaten des Kranken eine Schilderung der Krankheitserscheinungen, Diagnose und namentlich die Betonung der Gefährlichkeit oder 
Ungefährlichkeit des Kranken enthalten. Zeugnisse, welche im Inlande länger als 8, im Auslande länger als 24 Tage vor der Einlieferung ausgestellt wurden, dürfen nicht berücksichtigt werden.

§ 35 bestimmt jene Angehörige, welche berechtigt sind, um die Aufnahme von Geisteskranken anzusuchen, und bestimmt gleichzeitig den Begriff der Familie, wie ihn der zweite Abschnitt erfordert; solche Angehörige sind: Ehegemahl, Verwandte in auf- und absteigender Linie, inbegriffen die Geschwisterkinder, Adoptiveltern, Adoptivkinder, bei fehlender Geschäftsfähigkeit solcher deren gesetzlicher Vertreter, schließlich Vormund oder Kurator des Kranken. Im Notfalle kann der Anstaltsdirektor den Nachweis dieses Angehörigkeitsverhältnisses fordern.

Öffentliche Anstalten haben - soweit dies der Fassungsraum zuläßt - jeden Geisteskranken, ohne Rücksicht auf seine Zuständigkeit, aufzunehmen; nichtöffentliche kommunale Anstalten haben in erster Reihe nur die in die betreffende Kommune zuständigen Kranken aufzunehmen, andere bloß dann, wenn hierdurch die Aufnahme der ersteren nicht beeinträchtigt wird; die Aufnahme von Geisteskranken in nichtöffentliche private Anstalten ist dem Ermessen des Direktors anheimgestellt. Gefährliche Kranke können im Notfalle in der nächsten öffentlichen Anstalt provisorisch untergebracht werden.

Zu Lasten des Landeskrankenfonds können Kranke nur nach eingelangter ministerieller Erlaubnis aufgenommen werden; bei dringender Unterbringung gefährlicher Kranker kann der Direktor die Übernahme wegen mangelnder ministerieller Erlaubnis nicht verweigern, jedoch muß eine solche nachträglich erbeten werden.

Von der Übernahme eines Kranken muß der Direktor innerhalb 24 Stunden das dem Anstaltssitze nach zuständige Bezirksgericht, ferner Vormundschafts- und Aufsichtsbehörde verständigen. Das Bezirksgericht läßt den Kranken durch einen eigenen sachverständigen Arzt unverzüglich untersuchen; als solcher kann der Direktor oder ein Arzt der Anstalt bestimmt werden. Unmittelbar nach der Untersuchung spricht das Bezirksgericht beschlußweise aus, ob der übernommene Kranke aufgenommen werden kann. Im Falle eines Zweifels kann dem Sachverständigen ein Termin behufs eingehender Beobachtung bewilligt werden. Spricht das Bezirksgericht die Notwendigkeit der Aufnahme aus, kann gegen diesen Beschluß innerhalb 8 Tagen an den Gerichtshof, und wenn derselbe den Beschluß abändert, an die königl. Tafel appelliert werden. Diese Amtshandlungen sind nicht öffentlich.

Der fünfte Abschnitt, § 43-54, regelt die Entlassung aus der Irrenanstalt. Prinzipiell ist jeder in einer Irrenanstalt Internierte zu entlassen, wenn er sich als nicht geisteskrank erweist oder wenn seine Geisteskrankheit geheilt worden ist. Nicht gefährliche 
Kranke müssen über Wunsch eines Angehörigen entlassen werden, doch steht dem Anstaltsdirektor auch das Recht zu, nicht gefährliche Kranke ohne oder gegen den Willen der Angehörigen zu entlassen, wenn sich diese Maßregel als notwendig erweist. Kranke, welche über Aufforderung des Zivilgerichtes interniert wurden, können bloß über Beschluß des betreffenden Gerichtes entlassen werden. Gefährliche Kranke dürfen nicht entlassen werden, mit Ausnahme der Privatanstalten, wo der Direktor jeden Kranken entlassen kann, und über Wunsch eines berechtigten Angehörigen zu entlassen verpflichtet ist. Die Entlassung eines Kranken kann bloß sein gesetzlicher Vertreter fordern; ohne Einwilligung desselben und über Wunsch eines anderen Angehörigen darf der Kranke nur dann entlassen werden, wenn die zuständige Vormundschaftsbehörde einwilligt. In Ermanglung eines gesetzlichen Vertreters ist der Wunsch desjenigen Angehörigen maßgebend, über dessen Ansuchen der Kranke aufgenommen wurde. Im Falle einer Meinungsverschiedenheit oder über Wunsch einer interessierten Partei entscheidet die Vormundschaftsbehörde. Wird die Entlassung gegen den Rat des Direktors, oder (in Privatanstalten) bei bestehender Gefährlichkeit gewünscht, darf der Kranke bloß gegen Revers entlassen werden. Besteht Meinungsverschiedenheit über die eingetretene Heilung oder über die Gefährlichkeit, so entscheidet die die Aufnahme verfügende Gerichtsbehörde; in diesem Falle aber darf der Arzt der Anstalt nicht als Sachverständiger beigezogen werden. Die Entlassung kann vom Vertreter auch durch einen Bevollmächtigten verlangt werden, doch ist in diesem Falle eine beglaubigte Vollmacht erforderlich. Die Entlassung eines Kranken muß denselben Behörden angezeigt werden, wie die Übernahme des Kranken, mit Ausnahme des Bezirksgerichtes, und wird der Kranke nicht in geheiltem Zustande entlassen, muß auch die Aufsichtsbehörde erster Instanz verständigt werden. Wird aber der Kranke in eine andere Anstalt überführt, entfallen diese Meldungen. Gefährliche Kranke können auch behufs Überführung in eine andere Anstalt nur dann entlassen werden, wenn ihre Aufnahme dort gesichert ist.

Trifft der Direktor oder die Gerichtsbehörde keine Entscheidung bezüglich endgültiger Entlassung, so kann der Kranke auf bestimmte Zeit in häusliche Pflege gegeben, d. h. beurlaubt werden. Eine derartige Entlassung ist dann als endgültig zu betrachten, wenn während der vorher bestimmten Zeit weder der Kranke, noch seine Angehörigen, noch eine Behörde um die neuerliche Internierung ansuchen. Von einer vorläufigen Entlassung müssen die bei der Aufnahme vorgeschriebenen Behörden, und vom Ablauf der vorgeschriebenen Frist auch die Gerichtsbehörde, welche die provisorische Entlassung anordnet, verständigt werden. 
$\S 53$ bringt eine Neuerung in den Verfügungen bezüglich aus der Anstalt geflüchteter Geisteskranker. So steht dem Direktor das Recht zu, flüchtige Kranke durch die Polizei in die Anstalt zurückbringen zu lassen. Hält sich aber der geflüchtete nicht gefährliche Kranke an bekanntem Orte auf, und wird seine Überlieferung weder von kompetenten Angehörigen, noch von einer öffentlichen Behörde gefordert, darf seine gewaltsame Rückbeförderung in die Anstalt nicht erfolgen. Nach Ablauf von sechs Monaten nach erfolgter Flucht erlischt das Recht zur gewaltsamen Rückbeförderung unter allen Umständen. Jeder flüchtige Geisteskranke ist innerhalb 24 Stunden der Polizeibehörde anzumelden, unter Betonung dessen, ob der Kranke gefährlich oder nicht gefährlich ist.

Wird ein entlassener Kranker neuerdings einer Anstalt eingeliefert, sind dieselben Bestimmungen maßgebend wie bei der ersten Anstaltsaufnahme.

Im sechsten Abschnitt regelt $\S 55$ der Vollständigkeit halber den Transport von Geisteskranken; es hat, in Ermanglung anderen Übereinkommens oder statutenmäßiger Verfügungen, die Transportierung von Geisteskranken jene Behörde oder Person durchzuführen, welche die Einlieferung oder Entlassung fordert; erfolgt dies aber nicht innerhalb der vom Direktor festgestellten Frist, kann derselbe den Transport zu Lasten der betreffenden Person d:urchführen.

Der siebente Abschnitt regelt die überaus wichtige Frage der Beaufsichtigung der Geisteskranken. Diese war in Ungarn bisher im mehrfach erwähnten Gesetze XIV vom Jahre 1876 prinzipiell erwähnt, doch blieb die praktische Durchführung dieses Prinzipes zumeist nur am Papier, und namentlich waren die außerhalb der Irrenanstalten befindlichen Irren fast ohne jede Beaufsichtigung geblieben. Indem im vorliegenden Entwurfe gerade die Frage der Aufsicht der Geisteskranken eine eingehende und detaillierte, gesetzlich normierte Regelung und Durchführung erfährt, bietet der Entwurf nicht bloß eine große Zahl von Neuerungen, sondern bedeutet gleichzeitig einen gewaltigen Fortschritt in der Irrenfürsorge, dessen Wert und Bedeutung keiner eingehenden Betonung bedarf.

Dieser Abschnitt umfaßt die $\$ 56-79$ und gliedert sich in folgende vier Kapitel: Aufsichtsbehörsen, Beaufsichtigung der außerhalb der Irrenanstalten sich befindlichen Geisteskranken, Beaufsichtigung der Irrenanstalten und Evidenthaltung der Geisteskranken.

Kapitel I normiert überall drei Instanzen der A ufsich ts behörden, und zwar: in den Gemeinden: 1. den Gemeinde- oder Kreisarzt, 2. den Vizegespan des Komitates, 3. die Verwaltungskommission des Komitates. In den Städten mit geordnetem Magistrat: 1. der Bürgermeister mit 
dem Stadtphysikus, 2. und 3, wie bei den Gemeinden. In Städten mit autonomer Verwaltung: 1. der Stadthauptmann mit seinem Fachorgan, 2. der Bürgermeister mit dem städtischen Oberarzt, 3. der Verwaltungsausschuß der Stadt. In der Hauptstadt: 1. die Bezirksvorsteher mit dem Bezirksphysikus, 2. den Bürgermeister mit dem Oberphysikus, 3. den Verwaltungsausschuß. Jeder Verwaltungsausschuß hat eine Subkommission für das Irrenwesen zu entsenden, deren Vorsitzender der Vorsitzende des Ausschusses oder sein Vertreter ist, und deren Mitglieder der Oberphysikus der Stadtbehörde und ein vom Vorsitzenden auf die Dauer von drei Jahren ernanntes Mitglied des Verwaltungsausschusses sind. Den Organen der zwei ersten Instanzen obliegt die Beaufsichtigung der nicht in Irrenanstalten befindlichen Geisteskranken, während der Behörde dritter Instanz die Oberaufsicht dieser Geisteskranken, sowie die Aufsicht über die Irrenanstalten und die dort befindlichen Kranken zufällt. Die oberste Aufsichtsbehörde ist der Minister des Inneren, dessen Fachorgan der Landessanitätsrat ist, welcher eine Kommission für das Irrenwesen zu entsenden hat. Der Landessanitätsrat hat in allen Fragen von allgemeinem psychiatrischen Interesse auch ohne Aufforderung Vorschläge zu erstatten, begutachtet ihm vom Minister zugewiesene Fragen und beaufsichtigt über ministerielle Aufforderung die Irrenanstalten. Im Entwurfe sind die Obliegenheiten der Aufsichtsbehörden nur in großen Zügen gegeben, während die detaillierten Vorschriften den Ministerialverordnungen vorbehalten bleiben, da sich alle einzelnen Eventualitäten im Gesetze nicht vorsehen und angeben lassen.

Im Kapitel II wird die Beaufsichtigung der außerhalb der Anstalten befindlichen Geisteskranken normiert. Die behördliche Aufsicht erstreckt sich im Sinne des § 62 1. auf sämtliche in kommunaler Pflege befindlichen" Geisteskranken, 2. auf jene in Familienpflege sich befindlichen, a) deren Angehörige das Einschreiten der Behörde ansuchen, b) welche aus den' Irrenanstalten zeitweise oder dauernd entlassen wurden, und c) schließlich auf jene Personen, von welchen die Behörde in Erfahrung bringt, daß ihre Geisteskrankheit gefährlich ist, oder deren häusliche Pflege oder Behandlung als unzureichend erkannt wird. Nach $\S 63$ hat jede Person, welche für die Behandlung der Familienmitglieder oder Bediensteten zu sorgen hat, der Geisteskrankheit verdächtig erscheinende Personen entweder in eine Irrenanstalt einzuliefern oder der Aufsichtsbehörde anzumelden oder zu Hause pflegen zu lassen. Ist die häusliche Behandlung nicht durchführbar, so muß diesen Umstand der behandelnde Arzt der Aufsichtsbehörde anzeigen. Die Aufsichtsbehörde erster Instanz hat jeden ihr im Sinne der $\$ 82$ und 63 angemeldeten Geisteskranken durch ihr Fachorgan insbesonders auf geistigen Zustand und 
Gefährlichkeit untersuchen zu lassen, und muß das Nötige bezüglich Pflege, eventuell Unterbringung des Kranken verfügen. Über diese Untersuchung hat das Fachorgan innerhalb 24 Stunden Bericht zu erstatten, muß aber im Notfalle sofortige Verfügungen treffen. Erweist sich der untersuchte Kranke als gefährlich, oder die häusliche Pflege als undurchführbar, muß das untersuchende Fachorgan das notwendige Aufnahmezeugnis seiner Behörde unverzüglich vorlegen. Die Behörde selbst verfügt unverzüglich behufs zweckentsprechender Unterbringung des Kranken, längstens innerhalb 24 Stunden. Wird der Kranke auf eigene Kosten untergebracht, steht der Familie die freie Wahl der Anstalt zu. Dasselbe Verfügungsrecht steht auch den Aufsichtsbehörden höherer Instanz, der Polizeibehörde und auch anderen hierzu befugten Behörden zu.

Jede Aufsichtsbehörde niederer Instanz hat sämtliche in ihrem Aufsichtsrayon befindlichen Geisteskranken wenigstens einmal vierteljährlich überprüfen zu lassen, sorgt für die Beseitigung eventueller Mißstände, erstattet der höheren Behörde Vorschläge, und leitet bei eventuellen strafbaren Handlungen die strafrechtliche Verfolgung derselben ein. Utber Aufforderung der Aufsichtsbehörden höherer Instanz hat die ihr unterstehende Aufsichtsbehörde unverzüglich Bericht und Vorschläge zu erstatten.

Insolange ein gefährlicher Kranker nicht untergebracht werden kann, hat die Aufsichtsbehörde für seine und seiner Umgebung Sicherheit zu sorgen.

Kapitel III des siebenten Abschnittes regelt die Beaufsichtigung der Irrenanstalten. Der Entwurf macht in dieser Beziehung zwischen staatlichen und nicht staatlichen Anstalten blo $B$ insofern Unterschied, daß der Aufsichtsbehörde bei den staatlichen Anstalten, welche direkt dem Minister des Inneren unterstellt sind, nicht das Verfügungsrecht, sondern bloß das Recht Vorschläge zu erstatten zusteht. Nachdem die Beaufsichtigung der Irrenanstalten eine größere Routine und Erfahrung erheischt, weist der Entwurf diese Aufgabe der Aufsichtsbehörde dritter Instanz zu: dem Verwaltungsausschusse resp. dessen Subkommission für Irrenwesen, in welcher neben Laienelementen auch der Oberphysikus, also ein medizinischer Fachmann; ist.

Die Aufsichtsbehörse dritter Instanz hat nach $\S 72$ sämtliche in ihrem Rayon befindlichen Irrenanstalten jährlich einmal zu überprüfen, namentlich bezüglich Bau, Einrichtung, Zahl, Verpflegung und Unterbringung der Kranken; in den staatlichen Anstalten steht ihr bloß das Vorschlags-, in den anderen Anstalten auch sofortiges Verfügungsrecht zu. Diese Beaufsichtigung erstreckt sich auf sämtliche Lokalitäten, Kranke und Angestellte der Anstalt, und muß der Direktor oder sein Vertreter der Aufsichtsbehörde in allen Beziehungen unter- 
stützend und aufklärend an die Hand gehen. Nach $\S 73$ erstreckt sich aber das Aufsichtsrecht nicht auf die ärztliche Behandlung, ferner, bei staatlichen und privaten Anstalten, auf die Buchhaltung und Rechnungsgebarung. Die oberste Aufsichtsbehörde und das Ministerium des Inneren können die Anstalten sowohl von Amtswegen als auch über Ansuchen durch eine Aufsichtsbehörde oder durch Spezialorgane überprüfen lassen. Ähnliches Recht steht auch einem anderen Ministerium bezüglich der demselben unterstehenden Irrenanstalten zu.

Wenn in einem Aufsichtsorgane Zweifel bezüglich der Geisteskrankheit eines Anstaltsinsassen auftauchen, welche der Anstaltsdirektor zu zerstreuen nicht imstande ist, muß der nächst höheren Aufsichtsbehörde Meldung erstattet werden, welche das zuständige Waisenamt sofort verständigt; dieses sucht um die richterliche Überprüfung an, falls der fragliche Kranke keine Angehörigen besitzt oder dieselben keine Schritte unternehmen wollen.

Im IV. Kapitel dieses Abschnittes sind die Prinzipien der Evidenzhaltung der Geisteskranken festgelegt. Demnach hat die Aufsichtsbehörde erster Instanz sämtliche in den $\$ \$ 62$ und 63 bezeichneten Geisteskranken auf Grund der Meldung ihres Fachorganes, dann die nicht behördlicher Aufsicht unterstehenden Geisteskranken auf Grund der Meldung des behandelnden Arztes, schließlich die Anstaltskranken auf Grund der Meldung des Anstaltsdirektors in Evidenz zu halten, in welcher Liste sämtliche auf den Kranken bezüglichen Veränderungen einzutragen sind. Auf die Geisteskranken in Familienpflege erstreckt sich diese Evidenzhaltung nicht. Auf Grund der Listen der ersten Instanz hält die Aufsichtsbehörde dritter Instanz sämtliche Geisteskranke ihres Rayons in Evidenz, und legt diesen ihren Ausweis jährlich dem Ministerium des Inneren vor. (Die weiteren Details dieser Evidenzhaltung werden im Verordnungswege geregelt.)

Während in Ungarn bisher bloß Geisteskranke in Irrenanstalten aufgenommen werden durften, statuiert der achte Abschnitt des Entwurfes auch für Ungarn die Möglichkeit dessen, daß Kranke ohne äußeren Zwang, aus eigenem Willen freiwillig in Irrenanstalten aufgenommen werden können. Irrenanstalten können bloß mit ministerieller Genehmigung freiwillig eintretende Kranke (freiwillige Pensionäre) aufnehmen, zu welchem Behufe nichts anderes nötig ist als die notariell oder richterlich beglaubigte Erklärung des Eintretenden, daß er diesen Schritt aus eigenem Antriebe unternimmt und daß er sich dessen bewußt ist, daß ihm der Austritt jederzeit freigestellt bleibt; hierbei bleibt es dem Ermessen des Direktors anheimgestellt, ob er den freiwillig Eintretenden aufnimmt; Minderjährige oder unter Kuratel stehende Personen können nur mit Einwilligung des Vormundes aufgenommen werden. Freiwillig Eintretende müssen dem Bezirksgerioht 
nicht angemeldet werden, sondern der Anstaltsdirektor verständigt bloß den Vorsitzenden der Aufsichtsbehörde dritter Instanz vertraulich vom erfolgten Eintritte, weil sich Kapitel III des siebenten Abschnittes auch auf die freiwillig eintretenden Personen erstreckt; jede Verständigung über freiwillig Eintretende bildet Amtsgeheimnis. Derartige Kranke können jederzeit, auch gegen ihren Willen, entlassen werden; ihnen gegenüber darf keinerlei Zwang angewendet werden. Die Verfügungen des vierten und fünften Abschnittes finden auf freiwillig Eintretende keine Anwendung, doch treten dieselben sofort in Kraft, wenn der freiwillig Eintretende geisteskrank wird.

Der neunte Abschnitt regelt die Frage der Irrenanstaltsunterbringung solcher Geisteskranker, welche unter strafrechtlicher Untersuchung stehen. $\$ 86$ verfügt, daß Angeklagte, gegen welche die strafrechtliche Untersuchung wegen Geisteskrankheit aufgehoben resp. fallen gelassen wurde, durch die Staatsanwaltschaft der Aufsichtsbehörde erster Instanz unter Beifügung der Einstellungsbeschlüsse und Sachverständigengutachten angemeldet werden müssen, resp. Angeklagte, welche in Untersuchungshaft waren, dieser Behörde zu übergeben sind. Nach $\S 87$ geht die Aufsichtsbehörde in solchen Fällen genau so vor wie bei anderen Anmeldungen von Geisteskrankheit, bloß mit dem Unterschiede, daß die Untersuchung durch das Fachorgan der Behörde unterbleibt. Nach $\S 88$ sind für die Aufnahme solcher Kranker die Verfügungen des vierten Abschnittes in entsprechender Weise anzuwenden und diese Kranke von den übrigen möglichst abgesondert unterzubringen. Laut $\S 89$ des Entwurfes entscheidet bezüglich Entlassung derartiger Kranker nicht der Direktor, resp. die Gerichtsbehörde, sondern eine eigene Fachkommission des Ministeriums des Inneren. $\$ 90$ verfügt, daß die Bestimmungen dieses Abschnittes in entsprechender Weise auch auf jene Geisteskranke anzuwenden sind, welche nach Verbüßung ihrer Freiheitsstrafe oder im Verlaufe derselben geisteskrank geworden sind.

Im zehnten Abschnitte des Babarczi-Schwartzerschen Entwurfes findet sich eine überaus wichtige, auf Grund der neuesten psychiatrischen Anschauungen und Ansichten fußende Verfügung, nämlich Bestimmungen über geistig minderwertige Individuen. $\S 91$ besagt, daß in dauernd krankhaftem psychischen Zustande befindliche, nicht geisteskranke (psychisch minderwertige) Personen, bei welchen begründete Befürchtung besteht, daß sie auf freiem Fuße nicht bloß gegen das Vermögen gerichtete, strafrechtlich zu ahndende Verbrechen begehen können, in eigens zu diesem Behufe eingerichteten Anstalten unterzubringen und in denselben so lange zu belassen sind, als ihre Gemeingefährlichkeit oder ihr pathologischer Zustand besteht. $\$ \S 88$ und 89 beziehen sich auch auf solche Personen. 
Nach $\S 92$ des Entwurfes werden $\S 886$ und 87 auch dann anzuwendert sein, wenn sich Individuen nach Verbüßung ihrer Freiheitsstrafe alss geistig minderwertig erweisen.

Der elfte Abschnitt, $\$ \S 93-101$, enthält die Strafbestimmunge $n$ bei Umgehung der vorliegenden gesetzlichen Verfügungen. Mit Gefängnis bis zu 2 Monaten und Geldbuße bis zu 600 Kronen wird belegt, wer in ein nicht als Irrenanstalt errichtetes Krankenhaus unberechtigterweise Geisteskranke aufnimmt, wer nicht Geisteskranke in eine Irrenanstalt aufnimmt, und wer die gesetzliche Anmeldepflicht versäumt. Ein Vergehen verübt der Arzt, welcher behufs Unterbringung: einer geisteskranken Person in einer Irrenanstalt ein falsches Zeugnis: ausstellt, sowie auch derjenige, welcher wissentlich ein solches falsches. ärztliches Zeugnis benutzt oder annimmt; dieses Vergehen wird mit Freiheitsstrafe bis zu 2 Monaten geahndet. Mit Geldbuße bis zu 200. Kronen werden jene Anstaltsangestellten belegt, welche ihren Dienst gröblich verletzen, wenn dadurch Leben oder körperliche Sicherheit anderer gefährdet werden, oder aber, wenn sie die Flucht von Geisteskranken absichtlich erleichtern oder nicht verhindern. Mit Geldbuße bis zu 200 Kronen wird jene Person belegt, welche der im $\S 63$ vorgeschriebenen Anmeldepflicht innerhalb 3 Tagen nicht nachkommt, insofern für die Unterbringung des Geisteskranken nicht in andererWeise gesorgt wurde, und mit Geldbuße bis zu 100 Kronen der behandelnde Arzt, wenn er die ihm obliegende Meldung innerhalb 3 Tagen nicht erstattet. Sämtliche Vergehen gehören zur Kompetenz des Bezirksgerichtes. Die Verfügungen dieses Abschnittes finden keine Anwendung auf jene Vergehen, welche gleichzeitig gegen das Strafgesetzbuch verstoßen. Auch berühren diese Bestimmungen nicht die Disziplinarvorschriften der staatlichen, und Vertragsbestimmungen und Statuten der Privatirrenanstalten. Der Aufsichtsbehörde steht das Recht zu, Disziplinarstrafen zu verhängen, wenn in den nicht staatlichen Anstalten ihre auf Grund des vorliegenden Gesetzes erlassenen Verordnungen und Verbote mißachtet oder aber bezüglich sanitärer Gebarung erlassenen Aufforderungen nicht erfüllt werden. Diese Disziplinarstrafen sind entweder schriftlicher Verweis oder aber Geldbuße bis zu 100 Kronen.

Der zwölfte Abschnitt des Entwurfes, §§ 102-104, enthält die Durchführungsbestimmungen des Gesetzes. 